Publ. Mat. 61 (2017), 259-281

DOI: 10.5565 /PUBLMAT_61117_10

\title{
LOCALIZATION GENUS
}

\author{
JESPER M. MøLler AND JÉRÔME SCHERER
}

\begin{abstract}
Which spaces look like an $n$-sphere through the eyes of the $n$-th Postnikov section functor and the $n$-connected cover functor? The answer is what we call the Postnikov genus of the $n$-sphere. We define in fact the notion of localization genus for any homotopical localization functor in the sense of Bousfield and Dror Farjoun. This includes exotic genus notions related for example to Neisendorfer localization, or the classical Mislin genus, which corresponds to rationalization.
\end{abstract}

2010 Mathematics Subject Classification: Primary: 55S45; Secondary: 55R15, 55R70, 55P20, 22F50.

Key words: Genus, localization, Postnikov section, connected cover, completion, rationalization, self equivalence.

\section{Introduction}

Classically the genus of a nilpotent space $X$ of finite type, as introduced by Mislin in [19], consists of all homotopy types of nilpotent spaces $Y$ of finite type such that the localizations $Y_{(p)}$ and $X_{(p)}$ coincide at any prime $p$. That is, spaces in the same genus as $X$ cannot be distinguished from $X$ if one looks at them through the eyes of $p$-localization. Another analogous definition can be given in terms of rationalization and $p$-completions $[\mathbf{2 5}]$.

We introduce in this article the notion of localization genus. A localization functor $L$ in the category of spaces (or simplicial sets), as introduced by Bousfield [3] and Farjoun [9], is a homotopy functor equipped with a natural transformation $\eta$ from the identity which is idempotent up to homotopy. The study of such functors is motivated by the fact that it subsumes the notions of localization at a prime or a set of primes (e.g. rationalization) and $p$-completion, but also Postnikov sections, Quillen's plus-construction, and other nullification or periodization functors such

The first author is supported by the Danish National Research Foundation through the Centre for Symmetry and Deformation (DNRF92) and by Villum Fonden through the project Experimental Mathematics in Number Theory, Operator Algebras, and Topology. The second author is supported by FEDER/MINECO grant MTM2013-42293-P. 
as $P_{B \mathbb{Z} / p}$, which plays a central role in the Sullivan conjecture [18]. We write $\bar{L} X$ for the homotopy fiber of the natural map $\eta_{X}: X \rightarrow L X$. We define thus two genus sets associated to $L$ for any simply connected CW-space $X$ of finite type.

(1) The extended $L$ genus set for $X$ is the set $\bar{G}_{L}(X)=\{Y \mid L Y \simeq$ $L X, \bar{L} Y \simeq \bar{L} X\}$ of homotopy types $Y$ of $\mathrm{CW}$-spaces such that $L Y=L X$ and $\bar{L} Y=\bar{L} X$.

(2) The $L$-genus set for $X$ is the subset $G_{L}(X)$ of $\bar{G}_{L}(X)$ represented by $\mathrm{CW}$-spaces of finite type.

Our definition is suggested by the classical definition of the (completion) genus set [19], [25, Definition 3.5], and the extended (completion) genus set studied by McGibbon in [17]. We show in fact in Proposition 2.3 that when $L$ is rationalization, one gets back these classical notions. To illustrate our point of view we go through the computation of the extended rationalization genus $\bar{G}\left(S^{n}\right)$ of an odd sphere, Theorem 3.2, and we characterize in Corollary 3.4 those elements in $\bar{G}\left(S^{n}\right)$ corresponding to elements in the extended genus of the abelian group of integers, as studied by Hilton in [13].

To tackle technically harder problems we rely on Dwyer, Kan, and Smith's classifying space for towers of fibrations [8], a tool which has proven to be handy in similar situations [20]. This allows us in particular to do explicit computations of Postnikov genus sets for odd spheres and complex projective spaces.

Theorem 7.4. The extended Postnikov genus set $\bar{G}_{[n]}\left(S^{n}\right)$ of homotopy types of spaces $Y$ such that $Y[n] \simeq K(\mathbb{Z}, n)$ and $Y\langle n\rangle \simeq S^{n}\langle n\rangle$ is uncountable, in bijection with $\prod_{p} \mathbb{N}_{+}$, where the product is taken over all primes.

We also present in Section 5 a computation related to Neisendorfer's functor $[\mathbf{2 1}]$ and the Sullivan conjecture. The localization genus computations show combined features of the space one focuses on and the chosen localization functor. The notion of genus quantifies in which sense it is (not) sufficient to consider a given space locally, through the eyes of a localization functor $L$ and the associated fiber $\bar{L}$.

Acknowledgements. This project started during a visit of the first author at the Universitat Autònoma de Barcelona in 2008 and was rebooted during a visit of the second author at the Centre for Symmetry and Deformation in Copenhagen seven years later. We would like to thank the referee for their careful reading and many helpful suggestions. 


\section{Notation and background}

In this short section we fix our notation for spaces of maps and spaces of self-equivalences. We also introduce some terminology related to homotopical localization functors.

Let $X$ and $Y$ be (pointed) topological spaces. We are going to use the following notation throughout this note:

- $\operatorname{map}(X, Y)$ is the space of maps from $X$ to $Y$ and $\operatorname{map}(X, Y)_{*}$ the pointed mapping space;

- $\operatorname{aut}(X)$ is the topological monoid of self-homotopy equivalence of $X$ and $B \operatorname{aut}(X)$ is its classifying space;

- $\operatorname{Aut}(X)=\pi_{0}(\operatorname{aut}(X))$ is the group of homotopy classes of selfhomotopy equivalences of $X$;

- $\operatorname{aut}_{*}(X)$ is the topological monoid of based self-homotopy equivalences of $X$ and $B$ aut $_{*}(X)$ its classifying space.

The fibration $X \rightarrow B$ aut $_{*}(X) \rightarrow B$ aut $(X)$ is the universal $X$-fibration $[\mathbf{1 1}]$, so that every fibration sequence over a space $B$ with fiber $X$ arises from pulling back this fibration along a map $B \rightarrow B$ aut $(X)$. Let $X$ be a simply connected space. In that case the localization and completion constructions described below are well defined and classical.

- $X[n]$ is the $n$-th Postnikov approximation to $X$ and $X\langle n\rangle$ is the $n$-connected cover of $X$, for an integer $n \geq 1$;

- $X_{(p)}$ is the localization of $X$ at the prime $p, X_{0}$ its rationalization, and the torsion space $X_{\tau}$ is the homotopy fiber of the rationalization map $X \rightarrow X_{0}$;

- $X_{p}^{\wedge}$ is the $p$-completion of $X$ at the prime $p$ and the completion $X^{\wedge}$ is the product of all $p$-completions.

All these constructions can be made functorial in the category of (pointed) spaces $[\mathbf{9}]$ by using homotopical localization and cellularization functors.

Proposition 1.1. Let $X$ be a (pointed) simply connected $C W$-complex of finite type. There are homotopy equivalences of unpointed or pointed mapping spaces

$$
\begin{array}{rlrl}
\operatorname{map}\left(X_{\tau}, X_{\tau}\right) & \simeq \operatorname{map}\left(X^{\wedge}, X^{\wedge}\right), \operatorname{map}_{*}\left(X_{\tau}, X_{\tau}\right) & \simeq \operatorname{map}_{*}\left(X^{\wedge}, X^{\wedge}\right), \\
\operatorname{aut}\left(X_{\tau}\right) & \simeq \operatorname{aut}\left(X^{\wedge}\right), & \operatorname{aut}_{*}\left(X_{\tau}\right) & \simeq \operatorname{aut}_{*}\left(X^{\wedge}\right) .
\end{array}
$$

Proof: Completing the fibration $X_{\tau} \rightarrow X \rightarrow X_{0}$ yields a new fibration by the nilpotent fibration Lemma [4, II.4.8]. Hence $\left(X_{\tau}\right)^{\wedge} \simeq X^{\wedge}$, and Sullivan's arithmetic square $\left[4\right.$, VI.8.1] shows that $X_{\tau}$ is (also) the fibre 
of the rationalization map $X^{\wedge} \rightarrow\left(X^{\wedge}\right)_{0}$. Thus we have a natural completion map $X_{\tau} \rightarrow\left(X_{\tau}\right)^{\wedge} \simeq X^{\wedge}$ and applying the torsion functor to $X^{\wedge}$ yields an equivalence $\left(X^{\wedge}\right)_{\tau} \simeq X_{\tau}$.

Hence, we obtain two maps, by functoriality and continuity, see $[\mathbf{9}$, 1.C.8], namely $\operatorname{map}\left(X_{\tau}, X_{\tau}\right) \rightarrow \operatorname{map}\left(X^{\wedge}, X^{\wedge}\right)$ and $\operatorname{map}\left(X^{\wedge}, X^{\wedge}\right) \rightarrow$ $\operatorname{map}\left(X_{\tau}, X_{\tau}\right)$, which are homotopy inverses to each other. The same argument applies to pointed mapping spaces, as well as to spaces of self-equivalences.

Let $L$ be a homotopical localization functor, i.e. a coaugmented and idempotent homotopy functor in the category of spaces. It is sometimes more convenient to work in the Quillen equivalent category of simplicial sets, in particular when one needs models for mapping spaces. We will clearly say so when we do so. In practice localization functors arise as follows. To any map $f$ one associates a functor $L_{f}$ which inverts $f$ in a universal way, $[\mathbf{9}]$ and $[\mathbf{3}]$. Clever choices for the map yield homological localization, localization at a set of primes such as rationalization, Quillen's plus construction, Postnikov sections, etc.

The homotopy fiber of the coaugmentation $X \rightarrow L X$ is denoted by $\bar{L} X$. This yields an augmented functor, examples of which include the $n$-connected covers $X\langle n\rangle$ and torsion spaces $X_{\tau}$ mentioned above.

Definition 1.2 ([9, Definition 1.A.1]). Let $f$ be a map. A topological space $Y$ is $f$-local if $\operatorname{map}(f, Y)$ is a weak equivalence. When $f$ is of the form $A \rightarrow *$, an $f$-local space is called $A$-local (or $A$-null). This means that $Y=\operatorname{map}(*, Y) \rightarrow \operatorname{map}(A, Y)$ is a weak homotopy equivalence.

There exists an $f$-localization functor $L_{f}$ (or $A$-nullification functor $P_{A}$ when $f: A \rightarrow *$ ) with a natural transformation (coaugmentation) $\eta_{Y}: Y \rightarrow L_{f} Y$ such that $[9,1$. C.1]:

(1) $L_{f} Y$ is $f$-local for any space $Y$;

(2) the coaugmentation $\eta_{Y}: Y \rightarrow L_{f} Y$ is a weak homotopy equivalence when $Y$ is $f$-local;

(3) when the target $T$ is $f$-local the coaugmentation induces a homotopy equivalence $\operatorname{map}(Y, T) \leftarrow \operatorname{map}\left(L_{f} Y, T\right)$.

A map $g: Y \rightarrow Z$ is an $L_{f}$-equivalence if $L_{f} g: L_{f} Y \rightarrow L_{f} Z$ is a weak equivalence. The coaugmentation $\eta_{Y}: Y \rightarrow L_{f} Y$ is an $L_{f}$-equivalence and we will encounter other ones in this work.

We will also use a few basic facts about cellularization functors and cellular spaces. Let us fix a pointed space $A$. A space $B$ is $A$-cellular if it belongs to the smallest class of spaces containing $A$ and closed under weak equivalences and pointed homotopy colimits $[6]$. An $A$-cellular 
space $X$ is always killed by the $A$-nullification functor: $P_{A} X$ is contractible. Note also that if $B$ is $A$-cellular and $Y$ is $A$-local, then $Y$ is $B$-local as well due to the nice behavior of mapping out of a homotopy colimit.

\section{Genus and extended genus}

Let $L$ be a homotopical localization functor as introduced in the previous section. In this section we introduce the new concept of localization genus and explain how it is related to the classical notions of Mislin genus and complete genus.

Definition 2.1. Let $L$ be a localization functor and $X$ a simply connected $\mathrm{CW}$-complex of finite type.

- The extended L-genus set for $X$ is the set

$$
\bar{G}_{L}(X)=\{Y \mid L Y \simeq L X, \bar{L} Y \simeq \bar{L} X\}
$$

of weak homotopy types $Y$ of $\mathrm{CW}$-spaces such that $L Y=L X$ and $\bar{L} Y=\bar{L} X$.

- The L-genus set for $X$ is the subset $G_{L}(X)$ of $\bar{G}_{L}(X)$ represented by $\mathrm{CW}$-complexes of finite type.

The reason for this "generic" terminology comes from the relationship with the classical notion of genus. Recall that Mislin's definition [19] is given in terms of localization at primes: Two spaces $X$ and $Y$ belong to the same genus set if their localizations $X_{(p)}$ and $Y_{(p)}$ are homotopy equivalent at every prime $p$. This is a stronger requirement than merely asking for equivalent $p$-completions $X_{p}^{\wedge}$ and $Y_{p}^{\wedge}$, for any prime $p$, and equivalent rationalizations $X_{0}$ and $Y_{0}$, as shown for example by Belfi and Wilkerson in [2, Counterexample 2.1]. We will focus on the completion genus set as in [25, Definition 3.5] and the extended completion genus set $[\mathbf{1 7}]$.

Definition 2.2. Let $X$ be a simply connected CW-complex of finite type.

- The extended genus set of $X$ is the set $\bar{G}(X)$ of homotopy types of CW-complexes $Y$ such that $Y^{\wedge} \simeq X^{\wedge}$ and $Y_{0} \simeq X_{0}$.

- The genus set of $X$ is the subset $G(X)$ of $\bar{G}(X)$ represented by $\mathrm{CW}$-complexes $Y$ of finite type.

We show now that the classical completion genus coincides with our localization genus, when the chosen localization functor $L$ is rationalization. Since we restrict our attention to simply connected spaces here, 
one can choose the map $f$ to be the wedge of degree $p$ maps on the 2 -sphere, taken over all primes $p$. Then the Bousfield localization functor $L_{f}$ coincides with rationalization on simply connected spaces since local spaces are characterized by having uniquely $p$-divisible homotopy groups for any prime $p$.

Proposition 2.3. Let $X$ be a simply connected $C W$-complex of finite type. The extended rationalization genus set

$$
\bar{G}_{0}(X)=\left\{Y \mid Y_{0} \simeq X_{0}, Y_{\tau} \simeq X_{\tau}\right\}
$$

is the extended genus set $\bar{G}(X)$ and the rationalization genus set $G_{0}(X)$ coincides with the classical genus set $G(X)$.

Proof: We use the same idea as in the proof of Proposition 1.1 to show that $X_{\tau} \simeq Y_{\tau}$ if and only if $X^{\wedge} \simeq Y^{\wedge}$. This follows from the fact that $\left(X_{\tau}\right)^{\wedge} \simeq X^{\wedge}$, and because $X_{\tau}$ is (also) the fibre of $X^{\wedge} \rightarrow\left(X^{\wedge}\right)_{0}$.

Let us finally remark that all spaces in $G(X)$ are finite complexes when $X$ is a finite complex. Indeed, when $X$ is of finite type, the integral homology groups of any space in the genus set of $X$ are those of $X$. There is thus a Moore-Postnikov decomposition of such a space as successive homotopy cofibers of maps between (finite) Moore spaces, see for example [12, Chapter 8].

Example 2.4. Let $P$ be the nullification functor $[\mathbf{9}, 1$. A.4] with respect to the wedge $\bigvee B \mathbb{Z} / p$ taken over all primes $p$. Any finite complex is $B \mathbb{Z} / p$-null by Miller's solution to the Sullivan conjecture [18]. Hence there is a single space that looks like $X$ through the eyes of $P$ and $\bar{P}$ : $\bar{G}_{P}(X)=G_{P}(X)=\{X\}$.

We will elaborate on this in Section 5 by using Neisendorfer's Theorem for highly connected covers of finite complexes $[\mathbf{2 1}]$.

\section{The extended rationalization genus of an odd sphere}

In this section we turn our attention to a concrete example and propose an explicit computation of the rationalization genus for odd spheres. Let $n$ be an odd natural number. The extended rationalization genus set of the odd-dimensional sphere $S^{n}$ is according to [17, Theorem 2] an uncountable set. We offer an explicit description of this extended genus set and identify the elements known as pseudo-spheres. For this we will need some elementary abelian group theory.

Any torsion free abelian group $A$ of rank one can be seen, up to isomorphism, as a subgroup of $\mathbb{Q}$ containing $\mathbb{Z}$. For each prime $p$, let $h_{p}(A)=\max \left\{r \geq 0 \mid 1 \in p^{r} A\right\}$ denote the height of 1 at $p$. The height 
sequence of $A$ is the sequence $\chi(A)=\left(h_{p}(A)\right)_{p}$ of non-negative (or infinite) integers. Two sequences $\left(h_{p}\right)$ and $\left(k_{p}\right)$ are similar if the sum of the differences $\left|h_{p}-k_{p}\right|$ is finite. This means that the sequences differ in only a finite number of primes, and have $\infty$ in the same coordinates. A type is a similarity class of sequences. As explained in [10, Theorem 85.1], or [22, Theorem 10.47], isomorphism types of torsion free abelian groups of rank one are in bijection with types.

We start now with the fibration $S_{\tau}^{n} \rightarrow S^{n} \rightarrow\left(S^{n}\right)_{0}$ and will use that, since $n$ is odd, the rationalized sphere $\left(S^{n}\right)_{0}$ is an Eilenberg-Mac Lane space of type $K(\mathbb{Q}, n)$ as well as a Moore space of type $M(\mathbb{Q}, n)$. The torsion space $S_{\tau}^{n}$ is a Moore space $M(\mathbb{Q} / \mathbb{Z}, n-1)$. For any space $Y$ in the extended rationalization genus of $S^{n}$ we have a fibration sequence $M(\mathbb{Q} / \mathbb{Z}, n-1) \rightarrow Y \rightarrow K(\mathbb{Q}, n)$, the homotopy long exact sequence of which yields an exact sequence

$$
0 \rightarrow \pi_{n} Y \rightarrow \mathbb{Q} \stackrel{\partial}{\rightarrow} \mathbb{Q} / \mathbb{Z} \rightarrow \pi_{n-1} Y \rightarrow 0 .
$$

The idea is that the connecting homomorphism $\partial$ determines the homotopy type of $Y$.

Lemma 3.1. There is a bijection between isomorphism types of torsion free abelian groups of rank one and the double coset $\mathbb{Q}^{\times} \backslash \operatorname{Hom}(\mathbb{Q}, \mathbb{Q} / \mathbb{Z}) / \hat{\mathbb{Z}}$, where the units in $\mathbb{Q}$ act by pre-composition and the automorphisms $\hat{\mathbb{Z}}$ of $\mathbb{Q} / \mathbb{Z}$ by post-composition.

Proof: We define two maps. The first one, $\alpha: \operatorname{Hom}(\mathbb{Q}, \mathbb{Q} / \mathbb{Z}) \rightarrow\{A \mid$ $A$ torsion free abelian of rank one $\}$ sends a homomorphism $\partial$ to its kernel. The second one we call $\beta$. Given a subgroup $A$ of $\mathbb{Q}$, let $J$ be the subset of all primes consisting of those primes $p$ for which $h_{p}(A) \neq \infty$. Then the quotient $\mathbb{Q} / A$ is isomorphic to $\oplus_{p \in J} \mathbb{Z}_{p^{\infty}}$ and $\beta$ sends $A$ to the composite

$$
\mathbb{Q} \rightarrow \mathbb{Q} / A \cong \bigoplus_{p \in J} \mathbb{Z}_{p^{\infty}} \hookrightarrow \mathbb{Q} / \mathbb{Z} .
$$

Clearly $\alpha \circ \beta$ sends a torsion free abelian group of rank one $A$ to a subgroup of $\mathbb{Q}$ which is isomorphic to $A$. Moreover, given a homomorphism $\partial: \mathbb{Q} \rightarrow \mathbb{Q} / \mathbb{Z}$, the image $\beta(\operatorname{Ker} \partial)$ coincides with $\partial$ up to an isomorphism of $\mathbb{Q}$ (corresponding to the choice of an inclusion $\alpha(\partial) \subset \mathbb{Q}$ ) and an isomorphism of $\mathbb{Q} / \mathbb{Z}$ (corresponding to the choice of an isomorphism $\left.\mathbb{Q} / \operatorname{Ker} \partial \cong \oplus_{p \in J} \mathbb{Z}_{p^{\infty}}\right)$. This proves the lemma.

We proceed now with the construction of a space $Y(\partial)$ in the extended genus $\bar{G}\left(S^{n}\right)$ realizing any homomorphism $\partial: \mathbb{Q} \rightarrow \mathbb{Q} / \mathbb{Z}$ as a connecting map in the homotopy long exact sequence of the fibration 
$M(\mathbb{Q} / \mathbb{Z}, n-1) \rightarrow Y(\partial) \rightarrow K(\mathbb{Q}, n)$. We deduce from the bijection $[M(\mathbb{Q}, n-1), M(\mathbb{Q} / \mathbb{Z}, n-1)] \cong \operatorname{Hom}(\mathbb{Q}, \mathbb{Q} / \mathbb{Z})$ that there exists up to homotopy a unique map $\Delta: M(\mathbb{Q}, n-1) \rightarrow M(\mathbb{Q} / \mathbb{Z}, n-1)$ such that $\pi_{n-1}(\Delta)=\partial$. We define $Y(\partial)$ to be the homotopy cofiber of $\Delta$. We have therefore a cofibration sequence

$$
M(\mathbb{Q} / \mathbb{Z}, n-1) \rightarrow Y(\partial) \rightarrow M(\mathbb{Q}, n)
$$

which is seen to be a fibration sequence as well, for example by an elementary Serre spectral sequence argument. A complete characterization of such sequences that are simultaneously fibration and cofibration sequences has been obtained by Alonso [1], see also Wojtkowiak [26].

Theorem 3.2. The extended genus set $\bar{G}\left(S^{n}\right)$ is in bijection with the set of isomorphism classes of torsion free abelian groups of rank one.

Proof: Given a torsion free abelian group $A$ of rank one we get a homomorphism $\partial: \mathbb{Q} \rightarrow \mathbb{Q} / \mathbb{Z}$ from Lemma 3.1 and construct as above the space $Y(\partial)$. It realizes $\partial$ as connecting homomorphism in the homotopy long exact sequence, and its kernel is a group isomorphic to $A$. To show that we have indeed a bijection we must prove that the connecting homomorphism determines the homotopy type of $Y \in \bar{G}\left(S^{n}\right)$. Let $\partial$ be the connecting homomorphism for such a space $Y$ and let us compare $Y$ and $Y(\partial)$. We have a map $i: M(\mathbb{Q} / \mathbb{Z}, n-1) \rightarrow Y$ by definition of the extended genus set and consider $i \circ \Delta: M(\mathbb{Q}, n-1) \rightarrow Y$, where $\Delta$ is, as above, the unique map, up to homotopy, realizing $\partial$ on $\pi_{n-1}$. The map $\Delta$ factors through the Postnikov section $M(\mathbb{Q}, n-1) \rightarrow K(\mathbb{Q}, n-1)$, the second map being the homotopy fiber inclusion for $M(\mathbb{Q} / \mathbb{Z}, n-1) \rightarrow Y$. This shows that the composite $i \circ \Delta$ is homotopically trivial. Therefore the map $i$ factors through the homotopy cofiber of $\Delta$, i.e. $Y(\partial)$.

We have thus constructed a map $Y(\partial) \rightarrow Y$ which induces equivalences on rationalizations and torsion spaces. It is hence an equivalence as well.

In the final part of the section we restrict our attention to the $(n-1)$ connected members of the extended genus set of an odd sphere. We begin with a review of Hilton's investigations of the extended genus set of $\mathbb{Z}$ and groups of pseudo-integers $[\mathbf{1 3}, \mathbf{1 4}]$.

Definition $3.3([\mathbf{1 3}])$. A subgroup of the full rational group $\mathbb{Q}$ is a group of pseudo-integers if it contains $\mathbb{Z}$ but not $\mathbb{Z}[1 / p]$ for any prime $p$.

Since a group of pseudo-integers is a torsion free abelian group of rank one, in the terminology introduced at the beginning of the section, it is characterized by its type, which consists in only finite integers $h_{p}$. 
This subset of torsion free abelian group of rank one has been studied by Hilton in $[\mathbf{1 3}$, Theorem 2.3, 2.4].

According to $[\mathbf{1 3}$, Corollary 2.5], the extended genus set $\bar{G}(\mathbb{Z})$ of $\mathbb{Z}$, consisting of isomorphism classes of (not necessarily finitely generated) abelian groups $H$ such that $H \otimes \mathbb{Z}_{(p)} \cong \mathbb{Z}_{(p)}$ for all primes $p$, is the set of isomorphism classes of pseudo-integers. In other words, $\bar{G}(\mathbb{Z})$ is the set of isomorphism classes of torsion free abelian groups of rank one of $\infty$-free types $[\mathbf{1 0}, \S 85]$.

Corollary 3.4. The set of $(n-1)$-connected spaces in $\bar{G}\left(S^{n}\right)$ is in bijection with $\bar{G}(\mathbb{Z})$. The correspondence is given by $Y \mapsto \pi_{n}(Y)$.

Proof: The spaces in the extended genus of $S^{n}$ which are $(n-1)$-connected are characterized by the fact that the connecting homomorphism $\partial$ is surjective. In other words its kernel is a group of pseudointegers. We conclude by Theorem 3.2.

\section{A formula to compute localization genera}

In the previous section we have been able to establish a complete and explicit list of all homotopy types in the extended rationalization genus of an odd sphere. In general, for arbitrary spaces and arbitrary localization functors, this is not to be expected. Following the approach of Dwyer, Kan, and Smith in [8] to classify towers of fibrations, we propose in this section a formula which we use later on to perform computations of "Postnikov" and "Neisendorfer" genus. We start with the necessary background from $[\mathbf{8}]$.

Let $G$ be a space and consider the functor $\Phi$ which sends an object of Spaces $\downarrow B$ aut $(G)$, i.e. a map $t: X \rightarrow B$ aut $(G)$, to the twisted product $X \times{ }_{t} G$. Dwyer, Kan, and Smith describe a right adjoint $\Psi$ in $[8$, Section 4]. They find first a model for aut $(G)$ which is a (simplicial) group and thus acts on the left on $\operatorname{map}(G, Z)$ for any space $Z$. This induces a map $r: B$ aut $(G) \rightarrow B$ aut $(\operatorname{map}(G, Z))$. The functor $\Psi$ sends $Z$ to the projection map from the twisted product $B$ aut $(G) \times_{r} \operatorname{map}(G, Z) \rightarrow B$ aut $(G)$. This allows them right away to construct a classifying space for towers, in our case they will be of length 2.

Theorem 4.1 (Dwyer, Kan, Smith [8]). The classifying space for towers of the form $Z \stackrel{q}{\rightarrow} Y \stackrel{p}{\rightarrow} X$, where the homotopy fiber of $p$ is $G$ and that of $q$ is $H$, is $B$ aut $(G) \times_{r} \operatorname{map}(G, B$ aut $(H))$.

Remark 4.2. Working with the Dwyer-Kan-Smith model means that we deal with simplicial sets. The comparison with spaces is via the singular 
complex and geometric realization. Since the realization of a simplicial set is a CW-complex, all spaces we construct here have obviously the homotopy type of a CW-complex.

There is an interesting consequence of Theorem 4.1, which can be compared with our former result about the classifying space of the monoid of self-equivalences of a two-stage Postnikov piece [20, Theorem 5.3].

Theorem 4.3. Let $L$ be a localization functor and $X$ be a space such that $L \bar{L} X$ is contractible. There is then a bijection between $\bar{G}_{L}(X)$ and the set

$$
[L X, B \operatorname{aut}(\bar{L} X)] / \operatorname{Aut}(L X)
$$

of orbits for the action of the group $\operatorname{Aut}(L X)$ on the set $[L X, B \operatorname{aut}(\bar{L} X)]$.

Proof: Equivalence classes of towers of fibrations $Z \rightarrow Y \rightarrow *$ with successive fibres $L X$ and $\bar{L} X$ are classified by the set of path components of $B=B \operatorname{aut}(L X) \times_{r} \operatorname{map}(L X, B \operatorname{aut}(\bar{L} X))$. It is clear that any such space $Z$ fits in a fibration $\bar{L} X \rightarrow Z \rightarrow L X$, but we must show that the map $Z \rightarrow L X$ coincides with the localization map for $Z$. Fibrewise localization [9, Theorem 1.F.1] yields a natural transformation between fibration sequences

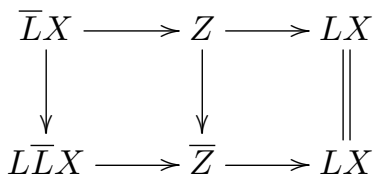

By assumption $L \bar{L} X$ is contractible and since by construction the map $Z \rightarrow \bar{Z}$ is an $L$-equivalence this proves that $L Z \simeq L \bar{Z} \simeq L X$.

A more symmetric, but completely equivalent formulation, is

$$
\bar{G}_{L}(X)=\operatorname{Aut}(\bar{L} X) \backslash[L X, B \operatorname{aut}(\bar{L} X)]_{*} / \operatorname{Aut}(L X) .
$$

The assumption that $L \bar{L} X$ is contractible is restrictive if we would require this for all spaces $X$. This would amount to imposing that the localization functor $L$ is a so-called nullification functor, i.e. a homotopical localization functor associated to a map of the form $A \rightarrow *$ such as a Postnikov section - when $A$ is a sphere $[\mathbf{9}, 1$. A.6] - or Quillen's plus construction. We impose this condition however on a single space, and this happens sometimes for localization functors that are not nullifications. When $X$ is a simply connected space of finite type and $L$ is rationalization, $p$-completion, or completion, then $L \bar{L} X \simeq *$. These are the main examples of interest in this note. 
According to Theorem 4.3, one can often compute an extended localization genus set via a Dwyer-Kan-Smith type formula.

Corollary 4.4. The classical extended genus set of a simply connected space $X$ of finite type is given by $\bar{G}(X)=\left[X_{0}, B \operatorname{aut}\left(X_{\tau}\right)\right] / \operatorname{Aut}\left(X_{0}\right)$.

\section{Postnikov genus and Neisendorfer's Theorem}

As a final computational example we will compute in the following sections the (extended) Postnikov genus of an odd sphere. We will rely on the Sullivan conjecture and the unexpected effect of the nullification functor $P_{B \mathbb{Z} / p}$ on highly connected covers of finite CW-complexes.

Let $P$ be the nullification functor with respect to the wedge $\bigvee B \mathbb{Z} / p$ taken over all primes $p$. Other functors that appear here are Postnikov sections $X \rightarrow X[N]$ and (highly) connected covers $X\langle N\rangle \rightarrow X$, aka nullification functors $P_{S^{N+1}}$ and homotopy fibers thereof $\bar{P}_{S^{N+1}}$. Hence, when we use a Postnikov section or a connected cover we will always use a functorial and continuous such construction. The following theorem is due to Neisendorfer and relies on Miller's solution to the Sullivan conjecture [18].

Theorem 5.1 ([21, Theorem 4.1]). Let $X$ be a simply connected finite complex with $\pi_{2}(X)$ finite and let $n \geq 1$ be a natural number. Then

$$
P(X\langle n\rangle)=\operatorname{holim}\left(X \rightarrow X_{0} \leftarrow X\left\langle n_{0}\right\rangle\right)
$$

so that $P(X\langle n\rangle)_{\tau} \simeq X_{\tau}$ and $P(X\langle n\rangle)^{\wedge} \simeq X^{\wedge}$.

For us the following consequences will be important. In particular point (2) has a similar flavor as Proposition 1.1 and will help us compute the monoids of self-equivalences which appear in the formula from Corollary 4.4 .

Proposition 5.2. Let $X$ be a simply connected finite complex with $\pi_{2}(X)$ finite. Suppose that $\pi_{>N}(X) \otimes \mathbb{Q}=0$ for some integer $N$. Then

(1) $P(X\langle N\rangle) \simeq X_{\tau}$.

(2) There are weak equivalences

$$
\operatorname{aut}_{*}(X\langle N\rangle) \simeq \operatorname{aut}_{*}\left(X_{\tau}\right) \simeq \operatorname{aut}_{*}\left(X^{\wedge}\right) \simeq \operatorname{aut}_{*}\left(X^{\wedge}\langle N\rangle\right)
$$

of topological monoids of pointed self homotopy equivalences.

(3) The obvious map $G(X) \rightarrow G(X[N])$ is a bijection whenever $\operatorname{aut}\left(X^{\wedge}\right) \rightarrow \operatorname{aut}\left(X[N]^{\wedge}\right)$ is surjective. 
Proof: Since $X\left\langle N_{0}\right\rangle$ is contractible we deduce from Theorem 5.1 that $P(X\langle N\rangle) \simeq X_{\tau}$, which proves (1). Hence starting with $X\langle N\rangle$ we see that we get first $X_{\tau}$ by applying $P$, then $X^{\wedge}$ by applying completion, and finally $X^{\wedge}\langle N\rangle \simeq X\langle N\rangle$ by taking the $N$-connected cover. We work here with pointed and continuous functors and obtain thus a chain of weak homotopy equivalences $\operatorname{aut}_{*}(X\langle N\rangle) \simeq \operatorname{aut}_{*}\left(X_{\tau}\right) \simeq \operatorname{aut}_{*}\left(X^{\wedge}\right) \simeq$ $\operatorname{aut}_{*}\left(X^{\wedge}\langle N\rangle\right)$. This shows $(2)$.

Wilkerson's double coset formula for the genus set [25, Theorem 3.8] exhibits $G(X)$ as double coset of the so-called $\pi_{*}$-continuous self-equivalences of $\left(X^{\wedge}\right)_{0}$ under the left action of aut $\left(X^{\wedge}\right)$ and the right action of aut $\left(X_{0}\right)$. Clearly $X$ and the Postnikov section $X[N]$ share the same rationalization and the same rationalization of their completions by assumption since $X\langle N\rangle$ is rationally trivial. Only the groups of selfequivalences of the completions might be distinct for $X$ and $X[N]$, but they act via rationalization so that the left action of $\operatorname{aut}\left(X^{\wedge}\right)$ factors through $\operatorname{aut}\left(\left(X^{\wedge}\right)_{0}\right) \simeq \operatorname{aut}\left(\left(X[N]^{\wedge}\right)_{0}\right)$. Thus, under the surjectivity assumption, the double cosets agree for $X$ and $X[N]$, which shows that the map $G(X) \rightarrow G(X[N])$ is bijective.

We note that $G(X[N])$ can be computed from Zabrodsky's exact sequence when $X_{0}$ is an $H$-space $[\mathbf{2 7}, \mathbf{2 8}],[\mathbf{1 7}$, Theorem 4].

Definition 5.3. The (extended) N-th Postnikov genus of a space $X$ is the (extended) localization genus set with respect to the nullification functor $P_{S^{N+1}}$. We write $G_{[N]}(X)$ and $\bar{G}_{[N]}(X)$ for these sets.

Hence a space $Y$ belongs to the extended genus $\bar{G}_{[N]}(X)$ if its $N$-th Postnikov section $Y[N]$ coincides with $X[N]$ and its $N$-connected cover $Y\langle N\rangle$ coincides with $X\langle N\rangle$.

Theorem 5.4. Let $X$ be a simply connected finite complex with $\pi_{2}(X)$ finite and suppose that $\pi_{>N}(X) \otimes \mathbb{Q}=0$ for some integer $N$. Assume moreover that $\operatorname{aut}\left(X^{\wedge}\right) \rightarrow \operatorname{aut}\left(X[N]^{\wedge}\right)$ is surjective. Then the only finite $C W$-complex in $G_{[N]}(X)$ is $X$.

Proof: If $Y$ belongs to $G_{[N]}(X)$, then $Y\langle N\rangle \simeq X\langle N\rangle$ and $Y[N] \simeq X[N]$. Thus, if $Y$ is finite, $Y^{\wedge} \simeq P(Y\langle N\rangle)^{\wedge} \simeq P(X\langle N\rangle)^{\wedge} \simeq X^{\wedge}$ by Theorem 5.1 and $Y_{0} \simeq Y[N]_{0} \simeq X[N]_{0} \simeq X_{0}$ showing that $Y \in G(X)$. But $X$ and $Y$ have the same image under the injective map $G(X) \rightarrow G(X[N])$ by Proposition 5.2(3), so $X \simeq Y$.

Example 5.5. The assumptions of Theorem 5.4 are fulfilled when $X$ is an odd sphere $S^{2 n+1}$ with $n \geq 1$. We only have to check that every self-equivalence of $K(\mathbb{Z}, n)^{\wedge}$ can be lifted to a self-equivalence of 
the completed sphere. But the former corresponds to a product of automorphisms of $\mathbb{Z}_{p}^{\wedge}$, any of which can be realized by a self-equivalence of $\left(S^{2 n+1}\right)_{p}^{\wedge}$.

Remark 5.6. Theorem 5.4 tells us that there are very few finite CW-complexes in a Postnikov genus set. However there are many infinite CWcomplexes of finite type in the extended genus set. For example when $X=S^{3}$ and $L$ is the third Postnikov section, the space $K(\mathbb{Z}, 3) \times S^{3}\langle 3\rangle$ is obviously in the $L$-genus of $S^{3}$. We will come back to this kind of example with a detailed computation in the next section.

Remark 5.7. It is not always true that there is a single finite complex in the Postnikov genus of a finite complex $X$. Let us consider for example the space $S^{2} \times S^{5}$ and the functor $L$ is chosen to be the second Postnikov section. Then $\left(S^{2} \times S^{5}\right)[2] \simeq K(\mathbb{Z}, 2)$ and $\left(S^{2} \times S^{5}\right)\langle 2\rangle \simeq S^{3} \times S^{5}$. It is easy to see that the space $\mathbb{C} P^{2} \times S^{3}$ also belongs to $G_{[2]}\left(S^{2} \times S^{5}\right)$. Of course the condition of the corollary are not fulfilled since neither $\pi_{2} S^{2}$, nor $\pi_{3} S^{2}$, are torsion.

It would be interesting to construct similar examples with higher Postnikov sections and at least 2-connected spaces, so that the $\pi_{2}$ assumption is trivially fulfilled.

\section{Self-equivalences of connected covers of a sphere}

Our next goal will be to determine the $n$-th Postnikov genus of an odd sphere $S^{n}$ with $n \geq 3$. This will be done by using Theorem 4.3, which involves the computation of the space of self-equivalences of the $n$-connected cover $S^{n}\langle n\rangle$. This section prepares the terrain for the genus computation in the next section and focuses on handy properties of $\operatorname{aut}\left(S^{n}\langle n\rangle_{p}^{\wedge}\right)$.

Since $S^{n}\langle n\rangle$ is a torsion space, it is weakly equivalent to the product of its $p$-completions $S^{n}\langle n\rangle_{p}^{\wedge}$. Now

$$
\begin{aligned}
\operatorname{map}\left(S^{n}\langle n\rangle, S^{n}\langle n\rangle\right) & \simeq \prod_{p} \operatorname{map}\left(S^{n}\langle n\rangle, S^{n}\langle n\rangle_{p}^{\wedge}\right) \\
& \simeq \prod_{p} \operatorname{map}\left(S^{n}\langle n\rangle_{p}^{\wedge} \times \prod_{q \neq p} S^{n}\langle n\rangle_{q}^{\wedge}, S^{n}\langle n\rangle_{p}^{\wedge}\right)
\end{aligned}
$$

But since $S^{n}\langle n\rangle_{p}^{\wedge}$ is $p$-complete and $\left(\prod_{q \neq p} S^{n}\langle n\rangle_{q}^{\wedge}\right)_{p}^{\wedge}$ is contractible, we see that this mapping space is weakly equivalent to $\prod_{p} \operatorname{map}\left(S^{n}\langle n\rangle_{p}^{\wedge}, S^{n}\langle n\rangle_{p}^{\wedge}\right)$. Therefore the subspace of self-equivalences also splits as a product

$$
\operatorname{aut}\left(S^{n}\langle n\rangle\right) \simeq \prod_{p} \operatorname{aut}\left(S^{n}\langle n\rangle_{p}^{\wedge}\right)
$$


The formula in Theorem 4.3 forces us to understand certain mapping spaces into $B$ aut $\left(S^{n}\langle n\rangle_{p}^{\wedge}\right)$. We start with a few elementary lemmas.

Lemma 6.1. The space $B$ aut $_{*}\left(\left(S^{n}\right)_{p}^{\wedge}\right) \simeq B \operatorname{aut}_{*}\left(S^{n}\langle n\rangle_{p}^{\wedge}\right)$ is $\Sigma B \mathbb{Z} / p^{k}$-local for any $k \geq 1$.

Proof: From Proposition 5.2(2) we know that the spaces of pointed self-homotopy equivalences of the $p$-completed sphere and its $n$-connected cover coincide. To prove the lemma we prove that the pointed mapping space $\operatorname{map}_{*}\left(\Sigma B \mathbb{Z} / p, B\right.$ aut $\left._{*}\left(\left(S^{n}\right)_{p}^{\wedge}\right)\right)$ is contractible. By the suspension-loop adjunction this mapping space is equivalent to $\operatorname{map}\left(B \mathbb{Z} / p\right.$, aut $\left._{*}\left(\left(S^{n}\right)_{p}^{\wedge}\right)\right)$. But aut ${ }_{*}\left(S^{n}\langle n\rangle_{p}^{\wedge}\right)$ consists of certain components of $\operatorname{map}_{*}\left(\left(S^{n}\right)_{p}^{\wedge},\left(S^{n}\right)_{p}^{\wedge}\right) \simeq \operatorname{map}\left(S^{n},\left(S^{n}\right)_{p}^{\wedge}\right)=\Omega^{n}\left(S^{n}\right)_{p}^{\wedge}$. All components of this iterated loop space have the same homotopy type, and they are $B \mathbb{Z} / p$-local by Miller's Theorem [18].

Lemma 6.2. The space $B \operatorname{aut}_{*}\left(\left(S^{n}\right)_{p}^{\wedge}\right) \simeq B \operatorname{aut}_{*}\left(S^{n}\langle n\rangle_{p}^{\wedge}\right)$ is $K(\mathbb{Z}[1 / p], 2)$ local.

Proof: Since $K(\mathbb{Z}[1 / p], 2)$ is $S^{2}[1 / p]$-cellular it is sufficient to prove that the above classifying space is $S^{2}[1 / p]$-local. We use the usual telescopic model for this localized sphere, i.e. the homotopy colimit of the diagram $S^{2} \stackrel{p}{\rightarrow} S^{2} \stackrel{p}{\rightarrow} \cdots$. Hence we have weak equivalences

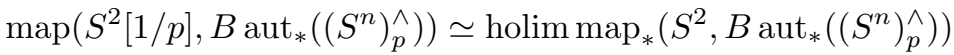

$$
\begin{aligned}
& =\operatorname{holim}_{\Omega} \operatorname{aut}_{*}\left(\left(S^{n}\right)_{p}^{\wedge}\right) \text {. }
\end{aligned}
$$

The homotopy groups of this inverse limit are all trivial since the tower we consider here consists of iterating multiplication by $p$ on finite $p$ groups (in particular all $\lim ^{1}$ terms vanish).

Zabrodsky's Lemma allows us to $\operatorname{mix} \bmod p$ and $p^{\prime}$-local information to deduce something integrally, compare this with Casacuberta's approach to Neisendorfer's Theorem in [5, Section 7].

Lemma 6.3. Fix $m \geq 2$. If a space $X$ is $K\left(\mathbb{Z}_{p^{\infty}}, m-1\right)$-local and $K(\mathbb{Z}[1 / p], m)$-local, then it is $K(\mathbb{Z}, m)$-local.

Proof: Since $X$ is $K\left(\mathbb{Z}_{p^{\infty}}, m-1\right)$-local, Zabrodsky's Lemma [7, Proposition 3.4] for the fibration $K\left(\mathbb{Z}_{p^{\infty}}, m-1\right) \rightarrow K(\mathbb{Z}, m) \rightarrow K(\mathbb{Z}[1 / p], m-1)$ produces an equivalence between the pointed mapping space $\operatorname{map}_{*}(K(\mathbb{Z}, m), X)$ and $\operatorname{map}_{*}(K(\mathbb{Z}[1 / p], m-1), X)$, which is assumed to be contractible. 
Even though we are not so sure whether $B$ aut $_{*}\left(S^{n}\langle n\rangle_{p}^{\wedge}\right)$ is $p$-complete, the previous lemmas allow us to understand a third and last local property.

Proposition 6.4. For any $m \geq 3$, the space $B$ aut $_{*}\left(S^{n}\langle n\rangle_{p}^{\wedge}\right)$ is $K(\mathbb{Z}, m)$ local. In particular, it is $K(\mathbb{Z}, n)$-local.

Proof: The space $K\left(\mathbb{Z}_{p^{\infty}}, m-2\right)$ is $\bigvee B \mathbb{Z} / p^{k}$-cellular, being a telescope of $K\left(\mathbb{Z} / p^{k}, m-2\right)$ 's which are cellular. Hence $K\left(\mathbb{Z}_{p^{\infty}}, m-1\right)$ is $\bigvee \Sigma B \mathbb{Z} / p^{k}$ cellular by the commutation rule of cellularization with respect to loop spaces [9, Theorem 3.A.2]. Lemma 6.1 implies that $B$ aut $_{*}\left(S^{n}\langle n\rangle_{p}^{\wedge}\right)$ is $K\left(\mathbb{Z}_{p^{\infty}}, m-1\right)$-local and Lemma 6.2 that it is $K(\mathbb{Z}[1 / p], m-1)$-local and hence $K(\mathbb{Z}, m)$-local by Lemma 6.3 .

We point out that the argument with a wedge is only necessary for $m=3$. For any larger value of $m$ we could have gone through the same proof with $\Sigma B \mathbb{Z} / p$.

We turn now to spaces of unpointed self-equivalences. Unlike their pointed analogues the classifying spaces $B$ aut $\left(\left(S^{n}\right)_{p}^{\wedge}\right)$ and $B$ aut $\left(S^{n}\langle n\rangle_{p}^{\wedge}\right)$ behave differently from the point of view of $K(\mathbb{Z}, n)$-nullification. The following proposition should certainly be compared to Zabrodsky's [29, Corollary C'], where he deals with locally finite homotopy groups in the source.

Proposition 6.5. The space $\operatorname{map}_{*}\left(K(\mathbb{Z}, n), B\right.$ aut $\left.\left(S^{n}\langle n\rangle_{p}^{\wedge}\right)\right)$ is homotopically discrete with $\mathbb{Z}_{p}^{\wedge}$ components.

Proof: Note first that $K(\mathbb{Z}, m)$ is $\Sigma K(\mathbb{Z}, 2)$-local for $m \geq 3$. For $m=3$, this is because the nullification $P_{\Sigma K(\mathbb{Z}, 2)} K(\mathbb{Z}, 3)$ is 1-connected with contractible loop space, $\Omega P_{\Sigma K(\mathbb{Z}, 2)} K(\mathbb{Z}, 3)=P_{K(\mathbb{Z}, 2)} K(\mathbb{Z}, 2) \simeq *[\mathbf{9}, 1$.G Proof of elementary facts, Theorem 3.A.1]. The case $m>3$ follows by induction starting at $m=3$ using $[\mathbf{9}$, Theorem 1.H.1] and the path fibration $K(\mathbb{Z}, m) \rightarrow * \rightarrow K(\mathbb{Z}, m+1)$.

Now that we know that $P_{\Sigma K(\mathbb{Z}, 2)} K(\mathbb{Z}, m) \simeq *$ for all $m \geq 3$, we also know that the implications

$\Omega B$ is $K(\mathbb{Z}, 2)$-local $\Longleftrightarrow B$ is $\Sigma K(\mathbb{Z}, 2)$-local $\Longrightarrow B$ is $K(\mathbb{Z}, m)$-local for $m \geq 3$

hold for any connected space $B$. 
There is a commutative diagram

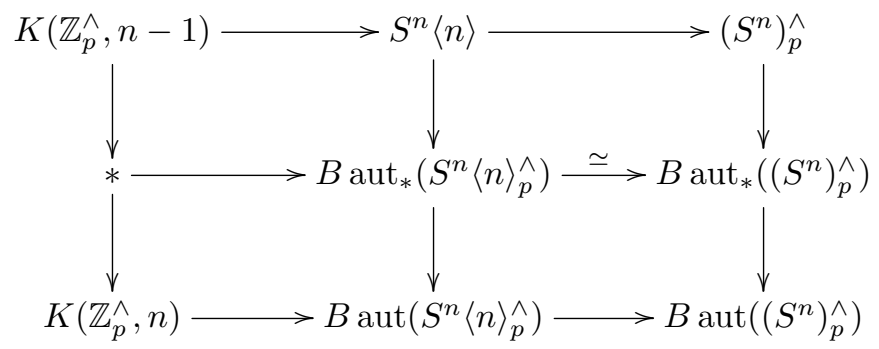

of fibrations. One of the maps here is a homotopy equivalence by Proposition 5.2(2). From the lower horizontal fibration we see that it suffices to show that $B$ aut $\left(\left(S^{n}\right)_{p}^{\wedge}\right)$ is $\Sigma K(\mathbb{Z}, 2)$-local. Equivalently, as we noted above, it suffices to show that aut $\left(\left(S^{n}\right)_{p}^{\wedge}\right)$ is $K(\mathbb{Z}, 2)$-local: In the evaluation fibration aut $_{*}\left(\left(S^{n}\right)_{p}^{\wedge}\right) \rightarrow \operatorname{aut}\left(\left(S^{n}\right)_{p}^{\wedge}\right) \rightarrow\left(S^{n}\right)_{p}^{\wedge}$, both the fibre, a disjoint union of $\Omega^{n}\left(S^{n}\right)_{p}^{\wedge}$, and the base space, $\left(S^{n}\right)_{p}^{\wedge}$, are $K\left(\mathbb{Z}_{p^{\infty}}, 1\right)$ local, which follows from Miller's theorem as stated for example in [23, Corollary 8.6.2] by an inductive argument as in [18, Theorem 10.1]. Since they are $p$-complete, both spaces are $K(\mathbb{Z}[1 / p], 2)$-local and we conclude by Lemma 6.3 that they are $K(\mathbb{Z}, 2)$-local. Then also the total space is $K(\mathbb{Z}, 2)$-local because every component is so by [9, Theorem 1.H.1].

\section{The extended Postnikov genus of an odd sphere}

We come now to our most sophisticated computation. We wish to determine the extended Postnikov genus $\bar{G}_{[n]}\left(S^{n}\right)$ when $n$ is odd. In other words, we wish to understand how many spaces $Y$ are extensions of $K(\mathbb{Z}, n)$ by $S^{n}\langle n\rangle$, i.e. how many spaces look like a sphere $S^{n}$ through the eyes of the $n$-th Postnikov section and the $n$-connected cover functors. We will sometimes call these spaces "fake spheres". We write $S_{p}^{n}$ for the fiberwise $p$-completion of $S^{n}$ sitting in the fibration $S^{n}\langle n\rangle_{p}^{\wedge} \rightarrow S_{p}^{n} \rightarrow K(\mathbb{Z}, n)$. By Theorem 4.3 and the preliminary remarks in the previous section we know that

$$
\begin{aligned}
& \bar{G}_{[n]}\left(S_{p}^{n}\right)=\left[K(\mathbb{Z}, n), B \operatorname{aut}\left(S^{n}\langle n\rangle_{p}^{\wedge}\right)\right] /\{ \pm 1\}, \\
& \bar{G}_{[n]}\left(S^{n}\right)=\left[K(\mathbb{Z}, n), \prod_{p} B \operatorname{aut}\left(S^{n}\langle n\rangle_{p}^{\wedge}\right)\right] /\{ \pm 1\},
\end{aligned}
$$

where we have identified $\operatorname{Aut}(K(\mathbb{Z}, n))$ with $\{ \pm 1\}$, and -1 acts on the integers by changing the sign. 
Theorem 7.1. The set of components of the unpointed mapping space $\operatorname{map}\left(K(\mathbb{Z}, n), B \operatorname{aut}\left(S^{n}\langle n\rangle_{p}^{\wedge}\right)\right)$ is $\mathbb{Z}_{p}^{\wedge} /\left(\mathbb{Z}_{p}^{\wedge}\right)^{\times}$. It is in particular an infinite, countable set. Hence, $\bar{G}_{[n]}\left(S_{p}^{n}\right)$ is in bijection with the set $\mathbb{N}_{+}$of natural numbers with a disjoint base point $*$.

Proof: The homotopy fiber of the evaluation

$$
\operatorname{map}\left(K(\mathbb{Z}, n), B \operatorname{aut}\left(S^{n}\langle n\rangle_{p}^{\wedge}\right)\right) \rightarrow B \operatorname{aut}\left(S^{n}\langle n\rangle_{p}^{\wedge}\right)
$$

is homotopically discrete and identifies with $\operatorname{Hom}\left(\mathbb{Z}, \mathbb{Z}_{p}^{\wedge}\right)$ by Proposition 6.5. Moreover the fundamental group

$$
\pi_{1} B \operatorname{aut}\left(S^{n}\langle n\rangle_{p}^{\wedge}\right) \cong \pi_{0} \operatorname{aut}\left(S^{n}\langle n\rangle_{p}^{\wedge}\right)
$$

coincides with $\pi_{0} \operatorname{aut}_{*}\left(\left(S^{n}\right)_{p}^{\wedge}\right) \cong\left(\mathbb{Z}_{p}^{\wedge}\right)^{\times}$, the $p$-adic units. Their action on the $p$-adic integers comes from the natural action on $\pi_{n}\left(S^{n}\right)_{p}^{\wedge}$. Thus the components of the mapping space we are looking at is the quotient $\mathbb{Z}_{p}^{\wedge} /\left(\mathbb{Z}_{p}^{\wedge}\right)^{\times}$.

Let $\mathbb{N}=\{0,1,2, \ldots\}$ be the set of natural numbers and $\mathbb{N}_{+}$the union of $\mathbb{N}$ with a disjoint base point $*$. The quotient $\mathbb{Z}_{p}^{\wedge} / \mathbb{Z}_{p}^{\times}$is in bijection with the set $\mathbb{N}_{+}$because any non-zero $p$-adic integer can be uniquely written as $p^{k} u$ where $k \in \mathbb{N}$ and $u$ is a unit [24, Chapter II, Proposition 2(b)]. The extended genus set has been identified as the quotient of this set under the action of \pm 1 . However, since -1 in $\mathbb{Z}$ is sent in the $p$-adics to a unit, there are no further identifications.

Construction 7.2. Here is an explicit way to construct the countable set $\bar{G}_{[n]}\left(S_{p}^{n}\right)$ of spaces $Y$ with $\pi_{n} Y \cong \mathbb{Z}$ and $Y\langle n\rangle \simeq S^{n}\langle n\rangle_{p}^{\wedge}$.

The fibration $S^{n}\langle n\rangle_{p}^{\wedge} \rightarrow S_{p}^{n} \rightarrow K(\mathbb{Z}, n)$ is classified by a map $c: K(\mathbb{Z}, n) \rightarrow$ $B \operatorname{aut}\left(S^{n}\langle n\rangle_{p}^{\wedge}\right)$. The proof of Theorem 7.1 shows that there is a bijection

$$
\mathbb{N}_{+} \rightarrow\left[K(\mathbb{Z}, n), B \operatorname{aut}\left(S^{n}\langle n\rangle_{p}^{\wedge}\right)\right] /\{ \pm 1\}
$$

taking $*$ to the constant map and the nonnegative integer $k \in \mathbb{N}$ to $c \circ p^{k}$.

Define the space $Y_{p, *}$ to be the product $S^{n}\langle n\rangle_{p}^{\wedge} \times K(\mathbb{Z}, n)$ and $Y_{p, k}$, for $k \in \mathbb{N}$, to be the homotopy pull-back of $K(\mathbb{Z}, n) \stackrel{p^{k}}{\rightarrow} K(\mathbb{Z}, n) \leftarrow S_{p}^{n}$, or, equivalently, the homotopy fibre of $S_{p}^{n} \rightarrow K(\mathbb{Z}, n) \rightarrow K\left(\mathbb{Z} / p^{k}, n\right)$, where the second map is reduction $\bmod p^{k}$. The bijection is then given by

$$
\begin{aligned}
\mathbb{N}_{+} & \longrightarrow \bar{G}_{[n]}\left(S_{p}^{n}\right) \\
k & \longmapsto Y_{p, k}
\end{aligned}
$$

We show now that one can detect which fake partially $p$-completed sphere one is considering by a simple cohomological computation. We will be more precise in the proof. 
Proposition 7.3. Ordinary cohomology distinguishes all elements in the extended Postnikov genus set $\bar{G}_{[n]}\left(S_{p}^{n}\right)$.

Proof: By Construction 7.2 these fake partially completed spheres fit into a tower of fibrations

$$
\cdots \rightarrow Y_{p, k+1} \stackrel{f_{k}}{\longrightarrow} Y_{p, k} \rightarrow \cdots \rightarrow Y_{p, 1} \stackrel{f_{0}}{\longrightarrow} Y_{p, 0}=S_{p}^{n}
$$

with fibers $K(\mathbb{Z} / p, n-1)$. Let $\iota_{k}$ denote a generator of $H^{n}\left(Y_{p, k} ; \mathbb{Z}\right) \cong \mathbb{Z}$, chosen in such a way that the image of $\iota_{k}$ under $f_{k}^{*}$ is $p \iota_{k+1}$.

At the prime 2 the algebra structure is sufficient to distinguish the fakes. Notice that the $\bmod 2$ reduction of $\iota_{1}$ is a polynomial generator detected in the mod 2 cohomology of $K(\mathbb{Z} / 2, n-1)$, but $\iota_{0}$ is an exterior generator. In general $\left(2^{k} \iota_{k}\right)^{2}=0$, but $\left(2^{k-1} \iota_{k}\right)^{2} \neq 0$. This shows that if $Y$ is any $(n-1)$-connected space with $\pi_{n} Y \cong \mathbb{Z}$ and $Y\langle n\rangle \simeq\left(S^{n}\langle n\rangle\right)_{2}^{\wedge}$, and $\iota$ denotes a generator of $H^{n}(Y ; \mathbb{Z})$, then $Y \simeq Y_{2, k}$ where $k$ is the smallest integer such that $\left(2^{k} \iota\right)^{2}=0$. If such an integer does not exist, then $Y \simeq Y_{2, *}$ for which the generator $\iota_{*}$ is polynomial.

At an odd prime $p$, the $\bmod p$ reduction of $\iota_{1}$ is an exterior generator detected in the $\bmod p$ cohomology of $K(\mathbb{Z} / p, n-1)$, but it has an integral Steenrod operation acting non-trivially on it. The pair $\left(\mathcal{P}^{1}, \beta \mathcal{P}^{1}\right)$ of admissible monomials in the $\bmod p$ Steenrod algebra yields a pair of elements in $H^{*}\left(K(\mathbb{Z}, n) ; \mathbb{F}_{p}\right)$ that are linked by a Bockstein, which corresponds in turn to a stable operation of order $p$ in $H^{n+2 p-1}(K(\mathbb{Z}, n) ; \mathbb{Z})$. Let us call it $\mathcal{P}^{1}$, as suggested by the identification done in [15]. In general this integral cohomological operation acts trivially on $p^{k} \iota_{k}$, but non-trivially on $p^{k-1} \iota_{k}$. This shows that if $Y$ is any space with $\pi_{n} Y \cong \mathbb{Z}$ and $Y\langle n\rangle \simeq\left(S^{n}\langle n\rangle\right)_{p}^{\wedge}$, and $\iota$ denotes a generator of $H^{n}(Y ; \mathbb{Z})$, then $Y \simeq Y_{p, k}$ where $k$ is the smallest integer such that $\mathcal{P}^{1}\left(p^{k} \iota\right)=0$. Again, if no such integer exists, then $Y$ is $Y_{p, *}$.

Theorem 7.4. The extended Postnikov genus set $\bar{G}_{[n]}\left(S^{n}\right)$ of homotopy types of spaces $Y$ such that $Y[n] \simeq K(\mathbb{Z}, n)$ and $Y\langle n\rangle \simeq S^{n}\langle n\rangle$ is uncountable, in bijection with $\prod_{p} \mathbb{N}_{+}$, where the product is taken over all primes.

Proof: We apply Theorem 4.3 and the identification $B \operatorname{aut}\left(S^{n}\langle n\rangle\right) \simeq$ $\prod_{p} B$ aut $\left(S^{n}\langle n\rangle_{p}^{\wedge}\right)$ obtained above. Theorem 7.1 shows that the set of unpointed homotopy classes $\left[K(\mathbb{Z}, n), B\right.$ aut $\left.\left(S^{n}\langle n\rangle\right)\right]$ is uncountable, in bijection with $\prod_{p} \mathbb{N}_{+}$. The action of the group $\operatorname{Aut}(K(\mathbb{Z}, n)) \cong\{ \pm 1\}$ is trivial at each prime since -1 is a unit in the $p$-adic integers, thus so is the action globally. 
Elaborating a little bit on Construction 7.2, one can construct explicitly all these fake spheres.

Construction 7.5. We identify $\left[K(\mathbb{Z}, n), B\right.$ aut $\left.\left(S^{n}\langle n\rangle\right)\right]$ with $\prod_{p} \mathbb{N}_{+}$. An element in this set is a sequence $K=\left(k_{p}\right)$ consisting either of a natural number or the base point $*$ for each prime $p$. For each such sequence consider the homotopy pull-back $Y_{K}$ of the diagram

$$
\prod_{p} Y_{p, k_{p}} \rightarrow \prod_{p} K(\mathbb{Z}, n) \stackrel{\Delta}{\longleftarrow} K(\mathbb{Z}, n)
$$

where the spaces $Y_{p, k_{p}}$ have been built in Construction 7.2, the first map is the product of $n$-th Postnikov sections, and the second arrow is given by the diagonal inclusion. The homotopy fiber of the map $Y_{K} \rightarrow K(\mathbb{Z}, n)$ is the product $\prod_{p} S^{n}\langle n\rangle_{p}^{\wedge} \simeq S^{n}\langle n\rangle$. For any prime $p$ the restriction to $B$ aut $\left(S^{n}\langle n\rangle_{p}^{\wedge}\right)$ yields $Y_{p, k}$ which is classified by $k_{p}$. This describes all spaces in $\bar{G}_{[n]}\left(S^{n}\right)$.

Thus we have a good handle on all these fake spheres $S^{n}$. What is so special about the good old $S^{n}$ among them? The answer is in Theorem 5.4, see also Example 5.5.

Proposition 7.6. Let $Y$ be a space such that $Y[n] \simeq K(\mathbb{Z}, n)$ and $Y\langle n\rangle \simeq S^{n}\langle n\rangle$. Then, if $Y$ is a finite complex, $Y$ has the homotopy type of $S^{n}$.

Finally we address the question of what happens when one changes the $n$-th Postnikov section for a higher one. The result will basically remain the same. An explicit computation would prove to be more difficult, but the concrete examples of fake spheres we have produced serve equally well now.

Proposition 7.7. Let $Y$ be an element in the extended Postnikov genus $\bar{G}_{[n]}\left(S_{p}^{n}\right)$, and $m \geq n$. For any large enough prime $p$ we have that $Y[m] \simeq S_{p}^{n}[m]$ and $Y\langle m\rangle \simeq S_{p}^{n}\langle m\rangle$.

Proof: Since $Y$ has been constructed so that $Y\langle n\rangle \simeq S^{n}\langle n\rangle_{p}^{\wedge}$, the same is true for a higher connected cover. The claim about the $m$-th Postnikov section follows by choosing $p>\frac{m-n+3}{2}$ so $\pi_{*} S^{n}$ has no $p$-torsion in degrees $<m$. 
This implies again that, for any $m$, there are uncountably many homotopy types of spaces which look like odd spheres through the eyes of the $m$-th Postnikov section and $m$-connected cover. We end the section with a related computation of the extended Postnikov genus of complex projective spaces.

Theorem 7.8. The extended Postnikov genus set $\bar{G}_{[2 n+1]}\left(\mathbb{C} P^{n}\right)$ is uncountable for any $n \geq 1$.

Proof: Let $C=\mathbb{C} P^{n}[2 n+1]$ be the $(2 n+1)$-st Postnikov section of $\mathbb{C} P^{n}$. The homotopy fiber of the inclusion $\mathbb{C} P^{n} \hookrightarrow \mathbb{C} P^{\infty}=K(\mathbb{Z}, 2)$ is the sphere $S^{2 n+1}$, so that we have fibration sequences $K(\mathbb{Z}, 2 n+1) \rightarrow C \rightarrow$ $K(\mathbb{Z}, 2)$ and $S^{2 n+1}\langle 2 n+1\rangle \rightarrow \mathbb{C} P^{n} \rightarrow C$ where $S^{2 n+1}\langle 2 n+1\rangle$ decomposes as $\prod_{p} S^{2 n+1}\langle 2 n+1\rangle_{p}^{\wedge}$. Obstruction theory shows that the $(2 n+9)$-st Postnikov section induces a bijective map $\left[\mathbb{C} P^{n}, \mathbb{C} P^{n}\right] \rightarrow[C, C]$ so that self-maps of $\mathbb{C} P^{n}$ and of $C$ are classified up to homotopy by their effect on $H^{2}(-; \mathbb{Z})$, see for example [16, Theorem 2.2]. In particular, $\operatorname{Aut}(C) \cong$ $\operatorname{Aut}\left(\mathbb{C} P^{n}\right) \cong \mathbb{Z}^{\times}=\{ \pm 1\}$ has two elements.

According to Theorem 4.3, $\bar{G}_{[2 n+1]}\left(\mathbb{C} P^{n}\right)$ is identified with the set $\left[C, B\right.$ aut $\left.\left(S^{2 n+1}\langle 2 n+1\rangle\right)\right] / \mathbb{Z}^{\times}$. Instead of computing this explicitly we will content ourselves with the more modest goal of showing that it is uncountable. Let $c: C \rightarrow B$ aut $\left(S^{2 n+1}\langle 2 n+1\rangle\right)$ be the classifying map for the standard $\mathbb{C} P^{n}$. We have seen that $B$ aut $\left(S^{2 n+1}\langle 2 n+1\rangle\right)$ splits as a product $\prod_{p} B$ aut $\left(S^{2 n+1}\langle 2 n+1\rangle_{p}^{\wedge}\right)$, and so the classifying map $c$ decomposes as a product $c=\prod_{p} c_{p} \circ \Delta$, where $\Delta: C \rightarrow \prod_{p} C$ is the diagonal map and $c_{p}: C \rightarrow B$ aut $\left(S^{2 n+1}\langle 2 n+1\rangle_{p}^{\wedge}\right)$. For any sequence $M=\left(m_{p}\right)_{p} \in \prod_{p} \mathbb{Z}$ of integers, let $P_{M}^{n}$ be the space classified by $\prod c_{p} \circ$ $\prod m_{p} \circ \Delta: C \rightarrow \prod B \operatorname{Aut}\left(X_{p}\right)$. For example $\mathbb{C} P^{n}$ and $C \times S^{2 n+1}\langle 2 n+1\rangle$ correspond respectively to the constant sequences (1) and (0).

Consider now the restriction of one of the components $c_{p} \circ m_{p}$ to the fiber $K(\mathbb{Z}, 2 n+1)$. The degree $m_{p}$ map on $\mathbb{C} P^{n}$ induces the degree $m_{p}^{n+1}$ map on the $2 n$-connected cover $S^{2 n+1}$, so that this restriction corresponds to the class of $m_{p}^{n+1}$ in the coset $\mathbb{Z}_{p}^{\wedge} /\left(\mathbb{Z}_{p}^{\wedge}\right)^{\times}$we have obtained in Theorem 7.1. In particular, choosing a non-invertible element $m_{p}=p^{k}$, this restriction represents a different homotopy class in $\left[K(\mathbb{Z}, 2 n+1), B \operatorname{Aut}\left(S^{2 n+1}\langle 2 n+1\rangle_{p}^{\wedge}\right)\right]$. In fact for any choice $k_{p} \in \mathbb{N}$, the sequences $\left(p^{k_{p}}\right)$ yield an uncountable number of homotopy types of fake complex projective spaces. Indeed the spaces $P_{\left(p^{k_{p}}\right)}^{n}$ are all distinct since the homotopy pull-back of $P_{\left(p^{k_{p}}\right)}^{n} \rightarrow C \leftarrow K(\mathbb{Z}, 2 n+1)$ is homotopy equivalent to the fake sphere described by the sequence $\left((n+1) k_{p}\right)$ as in Construction 7.5. 


\section{References}

[1] J. M. Alonso, Fibrations that are cofibrations. II, Proc. Amer. Math. Soc. 105(2) (1989), 486-490. DOI: 10.2307/2046968.

[2] V. Belfi and C. Wilkerson, Some examples in the theory of P-completions, Indiana Univ. Math. J. 25(6) (1976), 565-576.

[3] A. K. Bousfield, Constructions of factorization systems in categories, J. Pure Appl. Algebra 9(2) (1976/77), 207-220. DOI: 10.1016/0022-4049(77)90067-6.

[4] A. K. Bousfield and D. M. Kan, "Homotopy Limits, Completions and Localizations", Lecture Notes in Mathematics 304, Springer-Verlag, Berlin-New York, 1972.

[5] C. Casacuberta, Recent advances in unstable localization, in: "The Hilton Symposium 1993" (Montreal, PQ), CRM Proc. Lecture Notes 6, Amer. Math. Soc., Providence, RI, 1994, pp. 1-22.

[6] W. Chachólski, On the functors $C W_{A}$ and $P_{A}$, Duke Math. J. 84(3) (1996), 599-631. DOI: 10.1215/S0012-7094-96-08419-7.

[7] W. G. Dwyer, The centralizer decomposition of $B G$, in: "Algebraic Topology: New Trends in Localization and Periodicity" (Sant Feliu de Guíxols, 1994), Progr. Math. 136, Birkhäuser, Basel, 1996, pp. $167-184$.

[8] W. G. Dwyer, D. M. Kan, And J. H. Smith, Towers of fibrations and homotopical wreath products, J. Pure Appl. Algebra 56(1) (1989), 9-28. DOI: 10.1016/0022-4049 (89) 90119-9.

[9] E. D. Farjoun, "Cellular Spaces, Null Spaces and Homotopy Localization", Lecture Notes in Mathematics 1622, Springer-Verlag, Berlin, 1996. DOI: 10.1007/BFb0094429.

[10] L. Fuchs, "Infinite Abelian Groups. Vol. II", Pure and Applied Mathematics 36-II, Academic Press, New York-London, 1973.

[11] D. H. Gottlieb, The total space of universal fibrations, Pacific J. Math. 46 (1973), 415-417.

[12] P. Hilton, "Homotopy Theory and Duality", Gordon and Breach Science Publishers, New York-London-Paris, 1965.

[13] P. Hilton, On groups of pseudo-integers, Acta Math. Sinica (N.S.) 4(2) (1988), 189-192. DOI: 10.1007/BF02560598.

[14] P. Hilton, On the extended genus, Acta Math. Sinica (N.S.) 4(4) (1988), 372-382. DOI: 10.1007/BF02560641.

[15] S. O. Kochman, Integral cohomology operations, in: "Current Trends in Algebraic Topology, Part 1" (London, Ont., 1981), CMS Conf. Proc. 2, Amer. Math. Soc., Providence, R.I., 1982, pp. $437-478$. 
[16] C. A. McGibbon, Self-maps of projective spaces, Trans. Amer. Math. Soc. 271(1) (1982), 325-346. DOI: 10.2307/1998769.

[17] C. A. McGibbon, On the localization genus of a space, in: "Algebraic Topology: New Trends in Localization and Periodicity" (Sant Feliu de Guíxols, 1994), Progr. Math. 136, Birkhäuser, Basel, 1996, pp. 285-306.

[18] H. Miller, The Sullivan conjecture on maps from classifying spaces, Ann. of Math. (2) 120(1) (1984), 39-87. DOI: 10.2307/ 2007071.

[19] G. Mislin, The genus of an $H$-space, in: "Symposium on Algebraic Topology" (Battelle Seattle Res. Center, Seattle, Wash., 1971), Lecture Notes in Math. 249, Springer, Berlin, 1971, pp. 75-83.

[20] J. M. Møller AND J. Scherer, Can one classify finite Postnikov pieces?, Bull. Lond. Math. Soc. 42(4) (2010), 661-672. DOI: $10.1112 / \mathrm{blms} / \mathrm{bdq} 027$.

[21] J. A. NEISENDORFER, Localization and connected covers of finite complexes, in: "The Čech Centennial" (Boston, MA, 1993), Contemp. Math. 181, Amer. Math. Soc., Providence, RI, 1995, pp. 385-390. DOI: $10.1090 /$ conm/181/02044.

[22] J. J. Rotman, "An Introduction to the Theory of Groups", Fourth edition, Graduate Texts in Mathematics 148, Springer-Verlag, New York, 1995. DOI : 10.1007/978-1-4612-4176-8.

[23] L. Schwartz, "Unstable Modules over the Steenrod Algebra and Sullivan's Fixed Point Set Conjecture", Chicago Lectures in Mathematics, University of Chicago Press, Chicago, IL, 1994.

[24] J.-P. SERre, "A Course in Arithmetic", Translated from the French, Graduate Texts in Mathematics 7, Springer-Verlag, New York-Heidelberg, 1973.

[25] C. W. Wilkerson, Applications of minimal simplicial groups, Topology 15(2) (1976), 111-130. DOI: 10.1016/0040-9383(76)90001 $-\mathrm{X}$.

[26] Z. Wojtkowiak, On fibrations which are also cofibrations, Quart. J. Math. Oxford Ser. (2) 30(4) (1979), 505-512. DOI: 10.1093/ qmath/30.4.505.

[27] A. Zabrodsky, On the genus of finite CW- $H$-spaces, Comment. Math. Helv. 49 (1974), 48-64.

[28] A. Zabrodsky, $p$ equivalences and homotopy type, in: "Localization in Group Theory and Homotopy Theory, and Related Topics" (Sympos., Battelle Seattle Res. Center, Seattle, Wash., 1974), Lecture Notes in Math. 418, Springer, Berlin, 1974, pp. 161-171. 
[29] A. Zabrodsky, On phantom maps and a theorem of H. Miller, Israel J. Math. 58(2) (1987), 129-143. DOI: 10.1007/BF02785672.

Jesper M. Møller:

Matematisk Institut

Universitetsparken 5

DK-2100 København

E-mail address: moller@math.ku.dk

URL: htpp://www.math.ku.dk/ moller

Jérôme Scherer:

École Polytechnique fédérale de Lausanne

CH-1015 Lausanne

Switzerland

E-mail address: jerome.scherer@epfl.ch

$U R L:$ http://hessbellwald-lab.epfl.ch/Scherer

Primera versió rebuda el 6 de juliol de 2015, darrera versió rebuda el 4 de juliol de 2016. 